\title{
Critical residues involved in Toll-like receptor 4 activation by cationic lipid nanocarriers are not located at the lipopolysaccharide-binding interface
}

\author{
Caroline Lonez $^{1,2} \cdot$ Kate L. Irvine ${ }^{1} \cdot$ Malvina Pizzuto $^{2} \cdot$ Boris I. Schmidt ${ }^{2} \cdot$ Nick J. Gay $^{3}$ • \\ Jean-Marie Ruysschaert ${ }^{2} \cdot$ Monique Gangloff $^{3} \cdot$ Clare E. Bryant $^{1}$
}

Received: 16 February 2015/Revised: 31 March 2015/Accepted: 23 April 2015/Published online: 9 May 2015

(C) Springer Basel 2015

\begin{abstract}
DiC14-amidine is a cationic lipid that was originally designed as a lipid nanocarrier for nucleic acid transport, and turned out to be a Toll-like receptor 4 (TLR4) agonist as well. We found that while E. coli lipopolysaccharide (LPS) is a TLR4 agonist in all species, diC14-amidine nanoliposomes are full agonists for human, mouse and cat receptors but weak horse agonists. Taking advantage of this unusual species specificity, we used chimeric constructs based on the human and horse sequences and identified two regions in the human TLR4 that modulate the agonist activity of diC14-amidine. Interestingly, these regions lie outside the known LPS-binding domain. Competition experiments also support our hypothesis that diC14-amidine interacts primarily with TLR4 hydrophobic crevices located at the edges of the TLR4/ TLR4* dimerization interface. We have characterized potential binding modes using molecular docking analysis and suggest that diC14-amidine nanoliposomes activate TLR4 by facilitating its dimerization in a process that is myeloid differentiation 2 (MD-2)-dependent and cluster of differentiation 14 (CD14)-independent. Our data suggest
\end{abstract}

Electronic supplementary material The online version of this article (doi:10.1007/s00018-015-1915-1) contains supplementary material, which is available to authorized users.

Caroline Lonez

clonez@ulb.ac.be;

c1500@cam.ac.uk

1 Department of Veterinary Medicine, University of Cambridge, Cambridge, UK

2 Structure and Function of Biological Membranes, Université Libre de Bruxelles, Brussels, Belgium

3 Department of Biochemistry, University of Cambridge, Cambridge, UK that TLR4 may be activated through binding at different anchoring points, expanding the repertoire of TLR4 ligands to non-MD-2-binding lipids.

Keywords Toll-like receptor - Cationic lipid .

Nanoparticle $\cdot$ Activation mechanism $\cdot$ Species specificity

\section{Introduction}

Toll-like receptor 4 (TLR4) is a member of the innate immune system's Pattern Recognition Receptor (PRR) family, specialized in the recognition of bacterial lipopolysaccharides (LPS), components of the outer membrane of Gram-negative bacteria. The interaction between TLR4 and LPS requires two main co-receptors: the Myeloid Differentiation Factor 2 (MD-2), a glycoprotein physically associated with TLR4 on the cell surface and conferring to TLR4 its responsiveness to LPS and the Cluster of Differentiation 14 (CD14) which is believed to transport the LPS into the vicinity of TLR4/MD-2. Upon agonist recognition, TLR4/MD-2 homodimerizes and activates two main signalling pathways that depend on the adaptors recruited, both triggering the production of proinflammatory cytokines and chemokines: the Myeloid Differentiation primary response 88 (MyD88)-dependent cascade leading to the activation of the Nuclear Factor

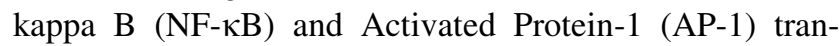
scription factors and the TIR-domain-containing adapterinducing interferon- $\beta$ (TRIF)-dependent pathway leading to the activation of the Interferon Regulatory Factor 3 (IRF-3) [1, 2].

The resolved structure of the dimeric complex TLR4/ MD-2 with bound LPS [3] has revealed that 5 acyl chains of the hexa-acylated Escherichia coli LPS (abbrv. E. coli 
LPS or EC-LPS) are buried deep inside a hydrophobic pocket in MD-2, with the 6th acyl chain partially exposed to the surface of the protein, participating in the dimerization interface. In contrast, lipid IVa, a tetra-acylated precursor of E. coli LPS, which is an antagonist in human, is completely buried inside the hydrophobic pocket of MD2 in a conformation that prevents TLR4 dimerization [4]. Depending on their structure (i.e. acylation pattern, number of phosphate groups), but also on the TLR4 and MD-2 mammalian species, LPS from different natural origins or synthetic LPS derivatives will bind and induce or prevent signalling with different efficiencies [5-8]. Penta-acylated lipopolysaccharide from Rhodobacter sphaeroides (RSLPS) acts as agonists of TLR4 in horses and hamsters, but as an antagonist in humans and mice [9-13]; lipid IVa, mentioned earlier, acts as an antagonist in human, but as an agonist in mouse, hamster, horse and cow [5, 9, 14-17]. Therefore, swapping experiments in which TLR4 from one species is used in combination with MD-2 from another species and inter-species chimera where amino acids found in one species are mutated with the corresponding residues from another species have allowed to identify regions in both TLR4 and MD-2 involved in species dependency of TLR4 ligands [14, 16, 18-20]. Recently, the comparison of the crystal structures of mouse TLR4/MD-2/lipid IVa (agonist) [17] and human MD-2/lipid IVa (antagonist) [4] confirmed the data obtained using species dependency and revealed that specific residues present in both mTLR4 and mMD-2 modulate the charge distribution of the complex, favouring the agonist positioning of lipid IVa in mouse TLR4 which promotes dimerization of mTLR4/MD-2/lipid IVa [17].

We showed previously that a cationic lipid synthesized in our laboratory, diC14-amidine [21, 22] (Fig. S1), activates TLR4 and MD-2-dependent MyD88 and TRIFdependent signalling pathways in human and murine dendritic cells [23-26]. DiC14-amidine's structure differs noticeably from the LPS structure (Fig. S1) both in size and charge, and aggregates into liposomes. We therefore hypothesized that the interaction mode of diC14-amidine nanoliposomes with TLR4/MD-2 would be different from traditional ligands such as LPS.

\section{Materials and methods}

\section{Constructs}

pcDNA3-hTLR4, pcDNA3-eTLR4, pcDNA3-hCD14, pEFIRES-hMD-2 and pEFIRES-eMD-2 were constructed as described earlier [16]. TLR4 chimeras were constructed by overlap extension PCR and point mutations were introduced by site-directed mutagenesis (QuickChange;
Stratagene) and mutations were confirmed by sequencing as described [16]. The ten different chimeras we used in this work are represented in Fig. S3: the first group corresponds to human TLR4 $(\mathrm{H})$ in which a specific region has been replaced by its corresponding equine insert $(\mathrm{E})$ to generate $\mathrm{HE}$ chimeras; the second group possesses the equine backbone $(\mathrm{E})$ which is locally replaced by human regions (EH chimeras). Constructs that failed to signal to LPS were excluded from this study.

\section{Materials}

DiC14-amidine was synthesized as described earlier [21] and stored as powder at $-20{ }^{\circ} \mathrm{C}$. Lipid films were formed by dissolving powder in chloroform, followed by solvent evaporation under nitrogen stream, vacuum drying overnight, and storage at $-20^{\circ} \mathrm{C}$. Before each experiment, lipid films were freshly resuspended in filtered Hepes $10 \mathrm{mM}$ heated at $55^{\circ} \mathrm{C}$ as previously described [22].

EC-LPS (UltraPure LPS-Escherichia coli O111:B4 subtype) and RS-LPS (Rhodobacter sphaeroides LPS) were obtained from InvivoGen and were freshly prepared for each experiment in water at a concentration of $1 \mathrm{mg} / \mathrm{ml}$ by vortexing, followed by sonication for $1 \mathrm{~min}$. LPS at $100 \mathrm{ng} / \mathrm{ml}$ corresponds approximately to $5-15 \mathrm{nM}$.

All cell culture media and components were purchased at Lonza.

\section{Cell culture and transient transfection}

Human embryonic kidney cells (HEK 293) were obtained from ATCC (Manassas, VA, USA). Cells were maintained in DMEM supplemented with $10 \%$ FCS, 2 mM L-glutamine, $100 \mathrm{U} / \mathrm{ml}$ penicillin and $100 \mu \mathrm{g} / \mathrm{ml}$ streptomycin. HEK293 cells were transfected as previously described [16]. Briefly, cells were seeded at $7.5 \times 10^{4}$ cells/well in a 96-well plate and transiently transfected 3 days later. Expression vectors containing cDNA encoding TLR4 (5 ng/ well), MD-2 (1 ng/well), and CD14 (1 ng/well), a NF-кB transcription reporter vector encoding Firefly luciferase (10 ng/well pNF-кB-luc; Clontech) and a constitutively active reporter vector encoding Renilla luciferase (5 ng/ well phRG-TK; Promega) together with empty vector ensure that an optimal amount of DNA was mixed with jetPEI (Polyplus transfection) according to the manufacturer's instructions.

After $48 \mathrm{~h}$ cells were stimulated for $6 \mathrm{~h}$ with diC14amidine (in serum-free DMEM), UltraPure LPS in complete medium, or $\mathrm{NiCl}_{2}$ (Sigma Aldrich) in complete medium. Cells were then washed with PBS and lysed with Passive Lysis Buffer (Promega). Luciferase and Renilla activity were then quantified on a FLUOstar Omega (BMG Labtech) using home-made luciferase reagent [20 mM 
Tricine, $2.67 \mathrm{mM} \mathrm{MgSO}_{4} .7 \mathrm{H}_{2} \mathrm{O}, 0.265 \mathrm{mM}\left(\mathrm{MgCO}_{3}\right)_{4-}$ $\mathrm{Mg}(\mathrm{OH})_{2} .5 \mathrm{H}_{2} \mathrm{O}, 0.1 \mathrm{mM}$ EDTA, $33.3 \mathrm{mM}$ DTT, $530 \mu \mathrm{M}$ ATP, $270 \mu \mathrm{M}$ Acetyl CoEnzyme A (Lithium salt), $470 \mu \mathrm{M}$ Luciferin (Biosynth), $\mathrm{pH} 7.8$, diluted 2 times in water before use] or coelenterazine (Biosynth) dissolved in ethanol at $1 \mathrm{mg} / \mathrm{ml}$ and diluted 500 times in PBS before use as described in [16]. Luciferase luminescence intensity was normalized to renilla luminescence intensity and data were expressed as fold induction as compared to non-induced control or as percentage as compared to LPS. Renilla luminescence serves as a control for experiment-inherent minor variations concerning cell numbers and transfection efficiencies between individual wells on the used microtiter plates. All transfected cells were tested for their ability to respond to EC-LPS in parallel to other ligands to ensure that the MD-2/TLR4 constructs were functional and to control any differences in protein expression efficiency.

For competition assays, $48 \mathrm{~h}$ after transfection, cells were incubated with RS-LPS for $1 \mathrm{~h}$, then cells were washed and stimulated as described. We used cells pretreated with RS-LPS before diC14-amidine stimulation or EC-LPS stimulation rather than co-administration, to prevent direct contact between lipids of opposing charges which could lead to possible interference within their respective micellar structures rather than at the level of receptor binding.

The THP1 cells were obtained from ATCC (Manassas, VA, USA) and were maintained in RPMI medium with $25 \mathrm{mM}$ HEPES supplemented with $10 \%$ FBS, $2 \mathrm{mM} \mathrm{L-}$ glutamine, $100 \mathrm{U} / \mathrm{ml}$ penicillin and $100 \mu \mathrm{g} / \mathrm{ml}$ streptomycin, $1 \mathrm{mM}$ sodium pyruvate and $20 \mu \mathrm{M}$ 2-Mercaptoethanol, at $37{ }^{\circ} \mathrm{C}$ at $5 \% \mathrm{CO}_{2}$.

For experiments, cells were primed with $10 \mathrm{nM}$ phorbol 12-myristate 13-acetate (PMA-Sigma Aldrich) to induce differentiation $28 \mathrm{~h}$ before stimulation. After this incubation period, the PMA-containing medium was removed and replaced with PMA-free complete medium for $4 \mathrm{~h}$ at $37{ }^{\circ} \mathrm{C}$ at $5 \% \mathrm{CO}_{2}$. Cells were then preincubated with the indicated amounts of neutralizing antibodies against human CD14 (InvivoGen) or Control antibody (InvivoGen) at a final concentration of $20 \mu \mathrm{g} / \mathrm{mL}$ for $1 \mathrm{~h}$, then stimulated with the indicated amounts of EC-LPS or diC14-amidine in serum-free medium (added in concentrated form into the antibody-containing medium to reach their final stimulant concentrations). After $4 \mathrm{~h}$ incubation, the supernatants were recovered and analysed by ELISA following manufacturer's instructions (DuoSet kits from R\&D Systems).

\section{Statistical analysis}

Multiple comparisons versus control group for each treatment within groups were made using One-Way ANOVA
(Holm-Sidak method) or Kruskal-Wallis One-Way Analysis of Variance on Ranks (Dunnett's method) when normality test failed, using SigmaPlot software.

\section{Computational methods}

\section{DiC14-amidine model}

The molecular structure of diC14-amidine was generated ab initio in Sybyl software version 8.1.1 (Tripos). The geometry of the lipid was optimized using the Powell minimisation method, with initial optimization based on the Simplex method, and with a gradient of $0.05 \mathrm{kcal} / \mathrm{mol}$ and a maximum of 100 cycles of iteration. Partial charges were computed based on the Gasteiger-Hückel charge method.

\section{TLR4 and MD-2 templates}

The molecular structures of the extracellular region of human TLR4 on its own and bound to MD-2 proteins as observed in the crystal structure of the LPS complex [3] were used for surface visualization and molecular docking of diC14-amidine molecules. Docking experiments were performed upon removal of the $E$. coli LPS ligands from the coordinate file.

\section{Molecular docking}

Autodock Vina software package [27] was used for docking diC14-amidine on TLR4-MD-2. The TLR4:MD-2 dimeric receptor complex was treated as a rigid protein complex. DiC14-amidine was fully flexible as its 30 torsion angles are within the maximum allowed limit. The Autogrid parameters were computed for the entire TLR4-MD-2 complex, with a grid sized $100 \times 100 \times 100 \AA^{3}$, but also for smaller areas centred on the regions shown to be important by mutagenesis, with a grid size of $40 \times 40 \times 40 \AA^{3}$. The grid was centred on the complex at $x=+12.322 ; y=-7.964$; $z=-5.891$. The default optimization parameters for the iterated local search global optimizer of Vina were used except for exhaustiveness, which was increased proportionally to the size of the grid (the default value of 8 was increased up to 32). Docking poses of the ligand were analysed and structural images were generated in PyMol (http:// www.pymol.org), Chimera [28], and LigPlot [29].

\section{Results}

\section{CD14 is not required in diC14-amidine activity}

To decipher the TLR4 activation mechanism induced by diC14-amidine, we wanted in a first step to determine the 
role of the co-receptors MD-2 and CD14 in the agonist activity of diC14-amidine. The importance of TLR4 and MD-2 was already demonstrated by the inability of $\mathrm{TLR}^{-/-}$and $\mathrm{MD}-2^{-/-}$bone marrow-derived dendritic cells to secrete IL-12p40 in response to diC14-amidine stimulation; however, the role of CD14 was not fully addressed so far [23]. We therefore transfected HEK293 cells with the plasmids coding for each protein in different combinations (Fig. 1a) and the cells were then stimulated with diC14-amidine liposomes or EC-LPS in the absence of serum.

As expected, in the absence of TLR4, MD-2 and CD14 no activation was observed for both ligands. Further, neither EC-LPS nor diC14-amidine was able to activate NF$\kappa B$ in the absence of MD-2 even in the presence of TLR4 and CD14 (Fig. 1a, right). However, while EC-LPS is unable to activate TLR4/MD-2 in the absence of CD14, diC14-amidine's TLR4 agonist activity was not affected by the lack of CD14, and was even found to have slightly increased.

We then evaluated CD14 requirement for NF- $\mathrm{KB}$ but also for IRF-3 induction on THP1 cells (by quantifying TNF- $\alpha$ and IP-10 in supernatants) by inhibiting CD14 using blocking antibodies (Fig. S2). This clearly confirms the non-requirement of CD14 for NF- $\mathrm{KB}$ activation by diC14amidine, but more surprisingly, stimulation of the cells with diC14-amidine also resulted in the secretion of hIP10 when CD14 was neutralized, demonstrating that the cationic lipid also does not require CD14 to trigger the TRIFdependent pathway.
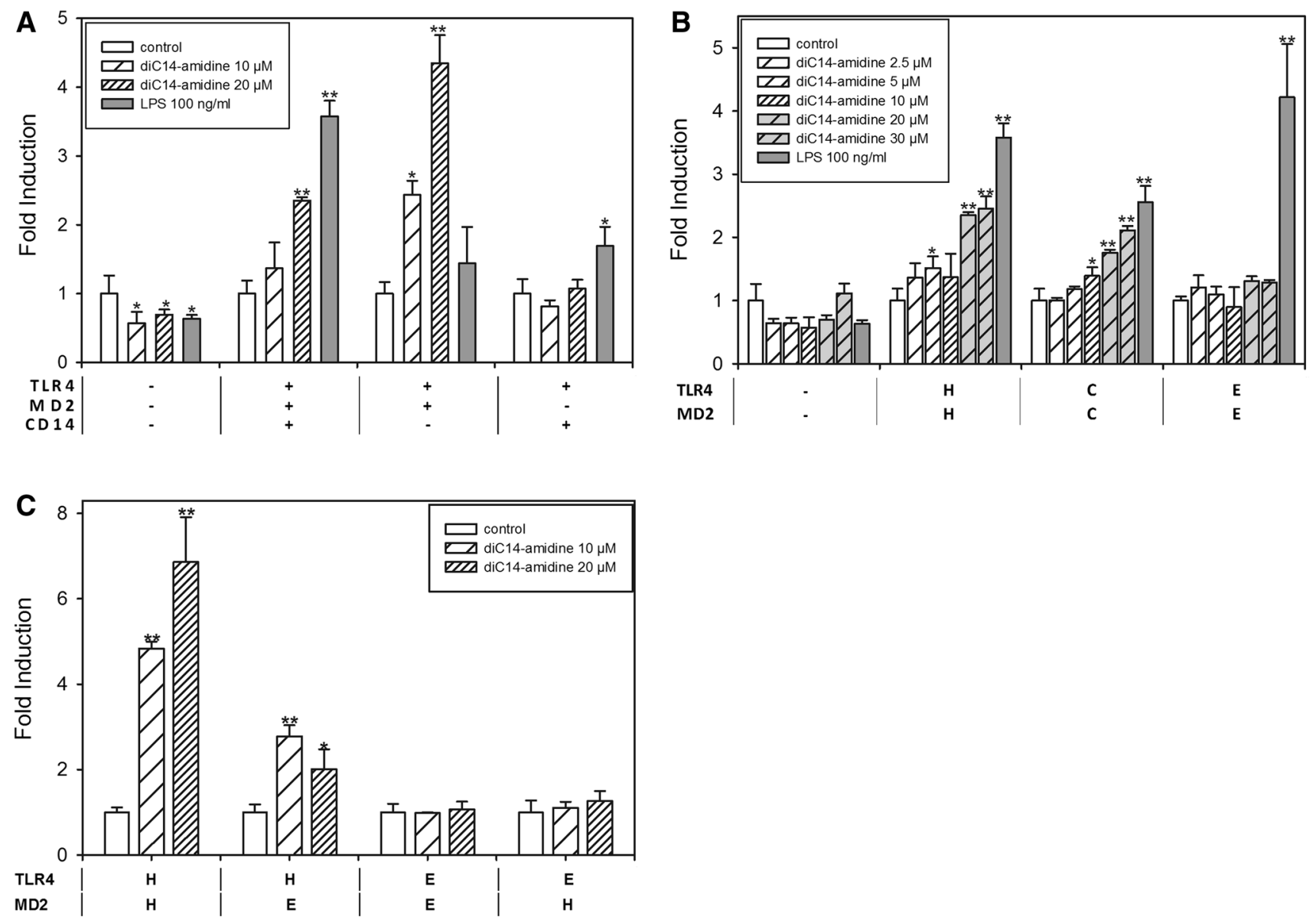

Fig. 1 Human TLR4 drives the activity of diC14-amidine. HEK 293 cells were transfected with plasmids encoding human TLR4 and/or MD-2 and/or CD14 (a) or TLR4 and MD2 from different species (b, c) with (b) or without (c) human CD14 together with firefly luciferase reporter plasmid dependent of NF- $\kappa \mathrm{B}$ activation. Two days after transfection, cells were stimulated for $6 \mathrm{~h}$ with diC14-amidine or LPS. Luciferase was then quantified in cell lysates. Data are represented as fold induction as compared to non-stimulated control

for each condition. Means are expressed \pm standard deviation with $n=3$. Representative of at least 2 independent experiments. $* p<0.05, * * p<0.01$ as compared to control (ANOVA). a Activation of NF- $\kappa \mathrm{B}$ by diC14-amidine requires both TLR4 and MD-2 but not CD14. b Species-dependent activity of diC14-amidine. $H$ human, $C$ cat, $E$ equine. c Partial activation of NF- $\kappa \mathrm{B}$ by diC14-amidine is maintained in the presence of human TLR4 


\section{Species-specific activity of diC14-amidine}

We previously showed that diC14-amidine is a TLR4 agonist in both human and mouse dendritic cells [23]. To understand the way diC14-amidine interacts with TLR4/ MD-2, we compared the TLR4 agonist activity of diC14amidine in two further species (cat and horse) by transfecting HEK293 cells with plasmids coding for TLR4, MD2 and CD14 from human (H), cat (C) and horse (E). We demonstrated (Fig. 1b) that, while EC-LPS is an agonist in all species, diC14-amidine is a full agonist for human and cat receptors, but induced low levels of activation in horse. Since we were unable to detect an antagonist effect of diC14-amidine on the full agonist ECLPS (data not shown), we therefore consider this compound to be a weak agonist in horse TLR4. This suggests that diC14-amidine is unable to induce signal transduction in the horse, while it binds and activates TLR4/MD-2 in other species.

\section{Horse MD-2 does not fully abolish diC14-amidine signalling activity, in contrast to horse TLR4, in inter-species assays}

To determine whether MD-2 or TLR4, or both, confer the observed species-specific differences in signalling, we conducted a series of MD-2/TLR4 swapping experiments (Fig. 1c). Cat TLR4/MD2, like human TLR4/MD2, was efficiently activated by diC14-amidine and comparative analysis between cat and human TLR4/MD2 was not likely to provide further information on how diC14-amidine interacts with this receptor complex. We focused therefore on human and horse comparisons. Whilst human TLR4 and MD-2 are fully activated by diC14-amidine nanoliposomes, and the equine TLR4 complex (eTLR4 + eMD-2) or the complex eTLR4 + hMD-2 are not, we still observed a partial activation of NF- $\kappa \mathrm{B}$ occurring by stimulating with diC14-amidine the combination of hTLR4 and eMD2 (Fig. 1c). In contrast, EC-LPS was able to fully activate all combinations of human and horse TLR4 and MD-2 (see Fig. S3).

The importance of TLR4 over MD-2 suggests a different mode of TLR 4 activation by diC14-amidine as compared to the more classical LPS derivatives. By comparison, similar experiments made with lipid IVa (agonist in horse and mouse but antagonist in human) showed that this ligand required both horse TLR4 and horse MD-2 or both mouse TLR4 and MD-2 to be active [16, 19] (no activation was found with combination of hTLR4/eMD-2 or eTLR4/ hMD-2) which was further confirmed by the crystal structures of mouse TLR4/MD-2/lipid IVa and human MD2/lipid IVa [4, 17]. Interestingly, another human TLR4 activator has been reported to mediate TLR4 signalling irrespectively of the origin of the transfected MD-2 coreceptor: nickel ions [30]. Schmidt and colleagues [30] proposed that nickel ions activate NF- $\kappa \mathrm{B}$ through binding of species-specific histidine residues in TLR4, triggering the formation of a TLR4/MD-2:TLR4*/MD-2* dimer that structurally resembles the one induced by LPS [30]. Although MD-2 is required for TLR4 dimerization, it does not participate in nickel binding explaining why species dependency of the agonist activity of nickel ions is solely dependent on human TLR4 [31].

\section{Antagonism of diC14-amidine activation of TLR4}

To determine whether the recognition interface of diC14amidine is different from the known LPS-binding site, we compared the effect of a TLR4 antagonist, Rhodobacter sphaeroides LPS (RS-LPS), on the TLR4 agonist activity of diC14-amidine and E. coli LPS (Fig. 2). RS-LPS (Fig. S1) is a potent antagonist for human TLR4, interacting with TLR4/MD-2 by inserting its lipid tails into MD-2's binding pocket [13].

Figure 2 shows the dose-response curves for EC-LPS $(B)$ and diC14-amidine $(A)$ upon pretreatment with increasing concentrations of RS-LPS. As expected, RS-LPS and EC-LPS compete for the same binding site, so the potency of the response to LPS was reduced after pretreatment with RS-LPS, but with no alteration of the maximal response reached at high concentrations of ECLPS (i.e. showing a parallel rightward shift of agonist dose-response curves). In contrast, RS-LPS decreases the potency and magnitude of the maximum response of diC14-amidine (RS-LPS effect cannot be negated, no matter how much diC14-amidine is present). This noncompetitive antagonism of RS-LPS on diC14-amidine's activity in contrast to the competitive antagonism seen for EC-LPS suggests that diC14-amidine binds at a different site on the TLR4/MD-2 complex. The same behaviour was found for nickel ions (Fig. 2c), underlining the similarities between these two TLR4 activators.

To identify the regions of TLR4 involved in the diC14amidine agonist activity, we tested the ability of human/ horse chimeras, in which regions of TLR4 from one species are exchanged with the corresponding ones from the other species, to be activated by diC14-amidine (Fig. 3a, b). This approach was previously used to identify residues in TLR4 and MD-2 that are important for the recognition of lipid IVa [14, 16, 19, 20] or RS-LPS [13] as TLR4 agonists and which were further confirmed by the crystal structures of human TLR4/MD2/EC-LPS, mouse TLR4/MD-2/lipid IVa and human MD-2/lipid IVa [3, 4, 17]. We constructed several chimeras first based on the regions known to be important in the case of lipid IVa or RS-LPS. Indeed, for these ligands, the LRR 14-18 region is critical for their 

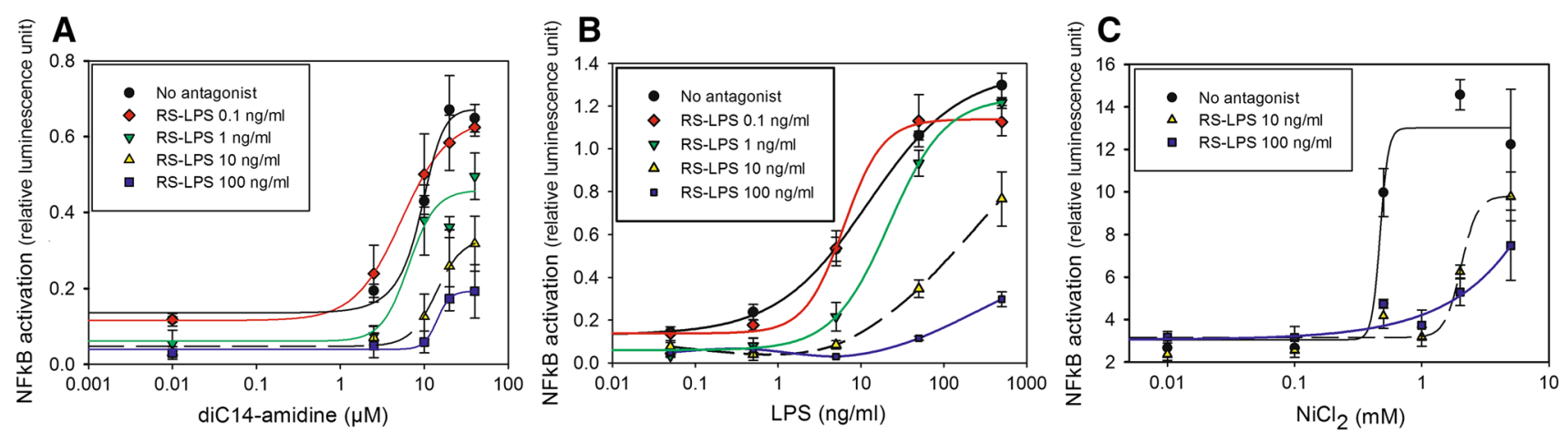

Fig. 2 Antagonist effect of RS-LPS on diC14-amidine, LPS and nickel TLR4 agonist activities. HEK 293 cells were transfected with plasmids encoding human CD14, MD-2, and TLR4, together with reporter plasmids. Two days after transfection, cells were pretreated with the indicated amount of RS-LPS for $1 \mathrm{~h}$, and then washed twice.

Cells were then stimulated with diC14-amidine (a), LPS (b) or $\mathrm{NiCl}_{2}$ (c), for $6 \mathrm{~h}$, and luciferase was quantified in cell lysates. Means are expressed \pm standard deviation with $n=3$. Representative of at least 2 independent experiments

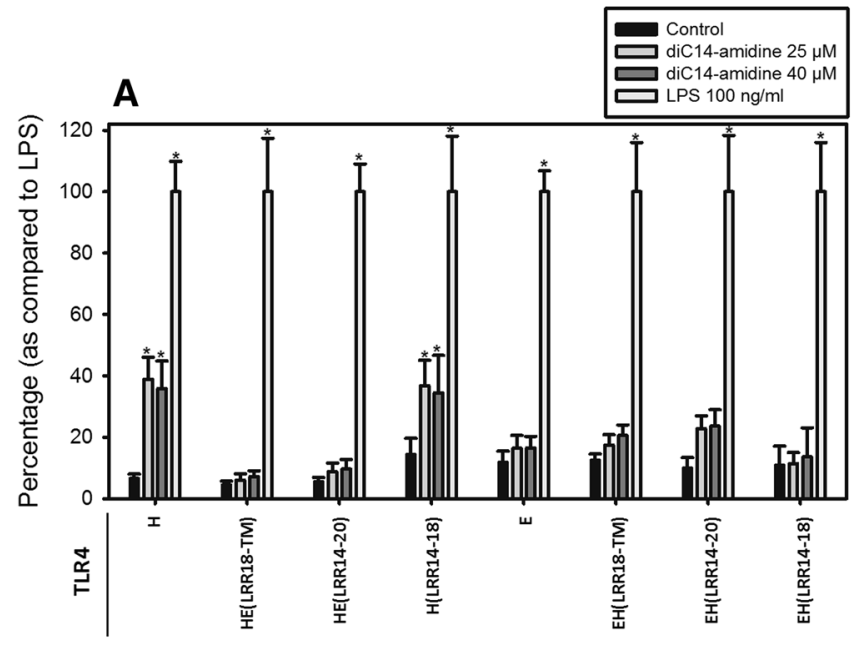

B

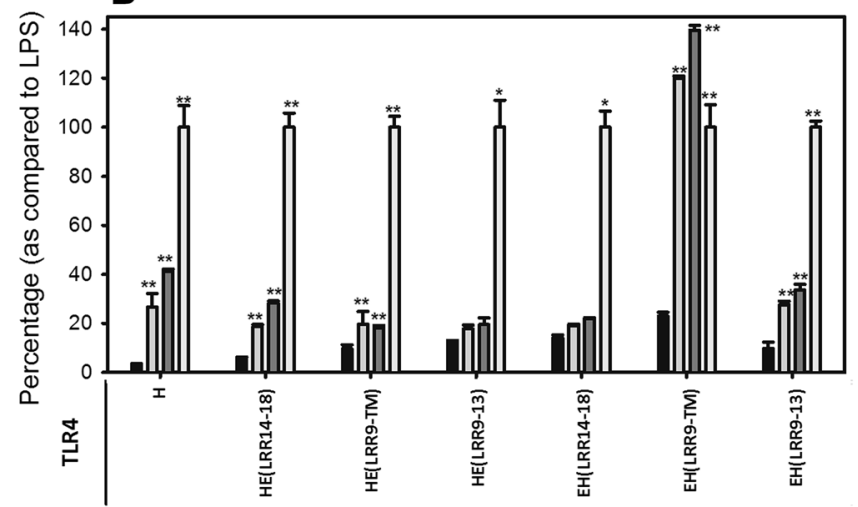

Fig. 3 TLR4 leucine-rich repeats LRR 9-13 and 18-20 are important for the activity of diC14-amidine. a, b, c HEK 293 cells were transfected with plasmids encoding human CD14, MD-2, and chimeric/mutant TLR4, together with reporter plasmids, then stimulated $48 \mathrm{~h}$ later with diC14-amidine for $6 \mathrm{~h}$ before quantification of NF- $\kappa \mathrm{B}$ activation. Luciferase was then quantified in cell lysates. Data are represented as fold induction as compared to non-stimulated

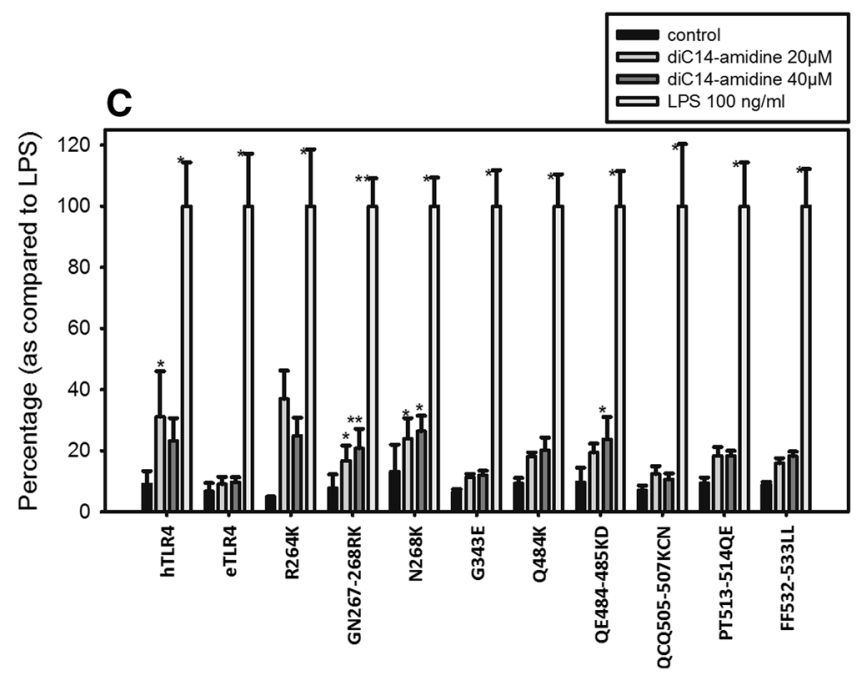

D

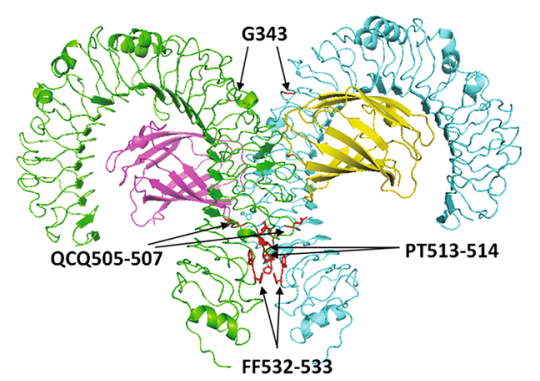

condition (control) for each species. Means are expressed \pm standard deviation with $n=3-15$. Representative of at least 2 independent experiments. $* p<0.05, \quad * * p<0.01$ as compared to control (ANOVA). d Localisation of the important residues for diC14amidine's agonist activity based on the known structure of TLR4/ MD-2/LPS [3] 
agonist activity in horse TLR4 and corresponds to the dimerization interface in the C-terminal domain interacting with one lipid chain of LPS [3]. A second region was also explored, corresponding to the primary binding interface (i.e. before ligand binding) between TLR4 and MD-2 located in the concave surface (LRR 9-13) of TLR4 [3] which is also known to interact with LPS derivative headgroups.

The chimeras HE (LRR 14-18) (human TLR4 with the equine LRR 14-18 insert), EH (LRR18-TM) (equine TLR4 with the human LRR 18-TM insert) and EH (14-20) (equine TLR4 with the human LRR 14-20 insert) are activated by diC14-amidine (Fig. 3a). Since these chimeras have only the human region LRR 18-20 in common (Fig. S4), this result suggests that residues 484-535 are critical for diC14-amidine recognition as an agonist. Another set of chimeras were also activated by diC14-amidine: HE (LRR 14-18) (already mentioned here above), EH (LRR9-TM) (equine TLR4 with the human LRR 9-TM insert) and EH (LRR9-13) (equine TLR4 with the human LRR 9-13 insert) (Fig. 3b), suggesting that a second region, corresponding to LRR 9-13 (see Fig. S4) (i.e. residues 252-370) is also involved in TLR4 agonist activity of diC14-amidine.

\section{Species-specific point mutagenesis identifies in TLR4 critical residues for the recognition of diC14- amidine}

The comparison of the human and equine sequences in the LRR 9-13 and 18-20 regions (Fig. S5) reveals that only 20 amino acids are different between horse and human in the LRR 18-20 region and 44 residues in the LRR 9-13 region. To identify the residues in human TLR4 important for diC14-amidine interaction, we produced several point mutations of human TLR4 whereby one or two residues were replaced by their equine homologues: R264K, GN267-8RK, N268K, G343E, Q484K, QE484-5KD, QCQ505-7KCN, PT513-4QE and FF532-3LL using site-directed mutagenesis, and tested their activity in response to diC14-amidine (Fig. 3c). Most mutants responded similarly to wild-type human TLR4 to diC14-amidine, while mutants FF532533LL and PT513-514QE showed a decreased activity in response to diC14-amidine and mutants G343E and QCQ505-7KCN were not activated by diC14-amidine, similar to equine TLR4. Interestingly, amino acids G343, Q505, Q507 and F533 do not interact with LPS in the crystal structure of TLR4/MD-2/LPS [3] but are important in TLR4-TLR4* interface interaction. The residues corresponding to Q507 and Q344 in horse (N508 and G345) are unique to this species (Fig. S5) and may therefore explain the difference of diC14-amidine's TLR4 agonist activity for horse as compared to other species.
It is striking that the mutated residues that lead to a loss (or a decrease) of the TLR4 agonist activity of diC14amidine are located in the TLR4-TLR4* dimerization interface (see Fig. 3d), while TLR4 residues involved in the interaction with the headgroup or the lipid chains of LPS [3] do not influence diC14-amidine's agonist activity. This strongly suggests that diC14-amidine interacts with TLR4 via a mechanism different to that previously proposed for LPS and its derivatives [3, 17]. Therefore, although the lipidic nature of diC14-amidine suggested that it was likely to interact with MD-2 and possess the so-called MD-2related lipid-recognition domain [32] to activate the TLR4 pathway, our pharmacological analysis and mutagenesis data now suggests that this is unlikely.

\section{TLR4 hydrophobic crevices are potential binding sites for diC14-amidine}

TLR4 possesses hydrophobic crevices that are spread all over its leucine and cysteine-rich regions with volumes up to $335 \AA^{3}$ according to CastP server calculations [33] (Fig. 4). We postulate, therefore, that diC14-amidine might be able to bind TLR4 through interaction with its hydrophobic crevices, with the possibility of binding several diC14-amidine molecules to several hydrophobic crevices throughout the TLR4-TLR4* interface. However, none of the hydrophobic grooves in TLR4 are as deep as those found in TLR2 which help to form TLR2-TLR1 and TLR2TLR6 heterodimers in the presence of bacterial tri- and diacylated lipopeptides (BLPs), respectively [34, 35]. BLPs are indispensable to hold these ectodomains together via hydrophilic and hydrophobic interactions. While two esterbound acyl chains are inserted into a pocket in TLR2, the amide-bound lipid of triacylated BLPs is fitted into a hydrophobic channel in TLR1. The latter is blocked off by Phe residues in TLR6 explaining the ligand specificity of the system $[34,35]$. To clarify the mode of action of diC14amidine, we generated docking models for this molecule in complex with the TLR4 ectodomain and the dimeric TLR4/ MD-2 complex [3]. DiC14-amidine could be docked in the vicinity of LRR19 residues Q505 and Q507 [at -5.2 k$\mathrm{cal} / \mathrm{mol}$ and with an apparent $\mathrm{K}_{\mathrm{i}}$ value in the micromolar range $(150 \mu \mathrm{M})]$. This putative binding site is located at the TLR4-TLR4* interface with the headgroup wedged between Q505 and Q507 and both myristate chains at the ascending flanks of TLR4 LRR 16-19 (Fig. 5). The latter Gln residue is conserved in human, mouse and cat and replaced by an Asn in horse TLR4. The shorter side chain in horse might explain the weaker activity of diC14-amidine in horse compared to other mammalian species. Therefore, our docking model proposes a potential binding site for diC14-amidine molecules in the proximity of the hydrophobic crevices found to be important in this study. 

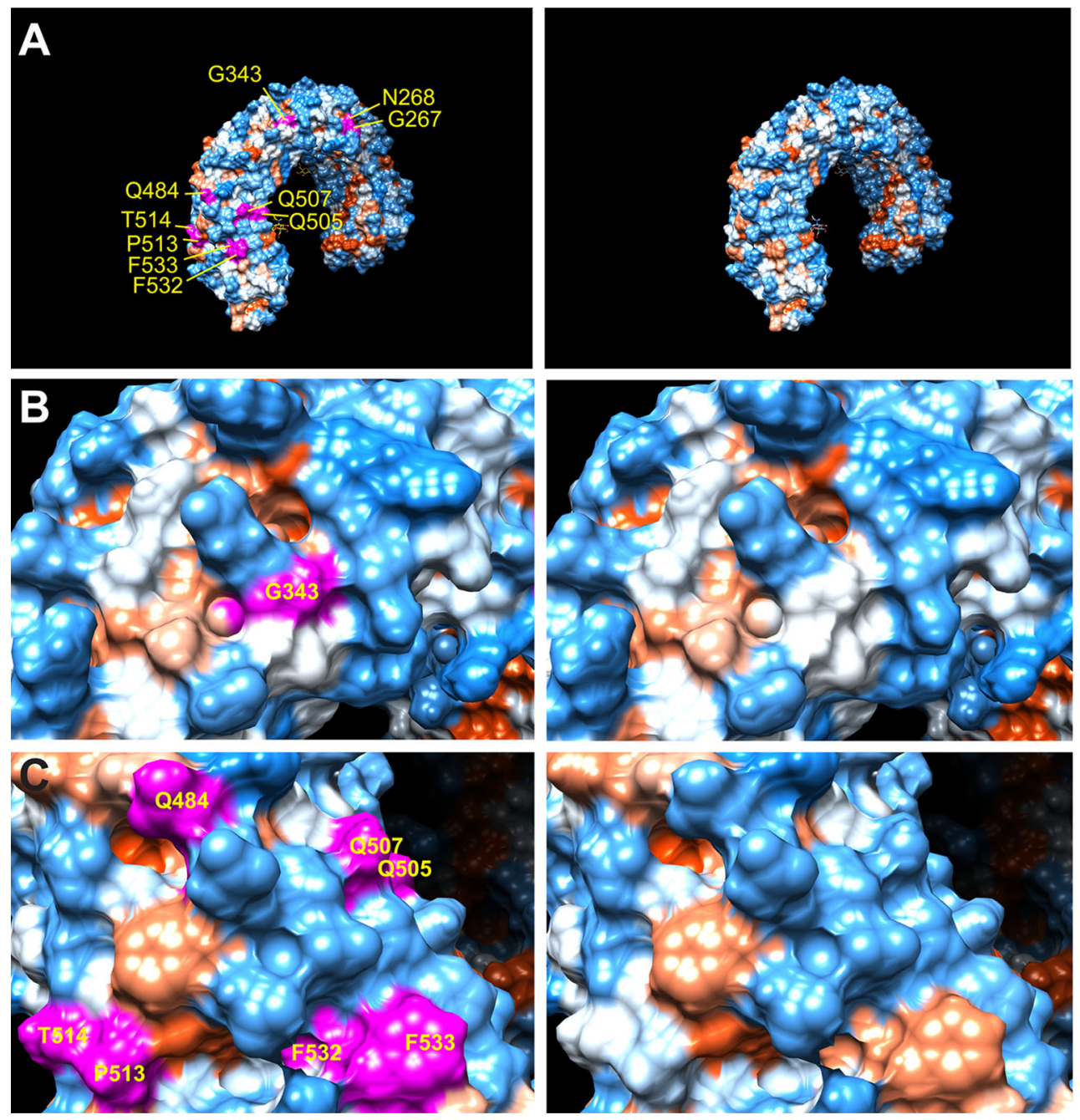

Fig. 4 Hydrophobic crevices on the surface of TLR4's ectodomain. a Molecular surface of the TLR4 ectodomain coloured according to its hydrophobicity (orange hydrophobic, blue hydrophilic). Speciesspecific residues that have been targeted by point mutagenesis are

highlighted in magenta in the left panels. b, c Close-up views illustrate the residues' proximity to hydrophobic crevices, potentially involved in diC14-amidine binding

\section{Discussion}

Here, we show how the cationic lipid diC14-amidine, a molecule initially designed for use as a lipid-based nanocarrier in gene therapy, is immunostimulatory. Our data suggest that diC14-amidine interacts directly with TLR4 and induces its dimerization via cross-linking two receptor chains. The subsequent cell signalling mechanism is analogous to the one that was proposed for TLR4 activation by nickel ions [30]. As already mentioned, it was previously proposed that nickel and cobalt ions trigger MD-2 dependent TLR4 dimerization and activation through chelation of species-specific histidine residues (H431, H456 and H458 on both TLR4 and TLR4*) located at the dimerization interface of human TLR4-TLR4 [30]. Oblak et al. [31] recently confirmed that nickel-binding site

is completely independent of the endotoxin-binding site but proposed that MD-2 nevertheless contributes to the interaction by providing supporting hydrophobic interactions with TLR4 which stabilize the TLR4/MD-2/Ni2+ complex in a proper conformation for cellular activation.

Cross-linking of TLR4 ectodomains, resulting in their activation, may also occur following incubation of cells with antibodies directed against TLR4 [36, 37]. This implies that different anchoring points can lead to dimerization and foresees other possible ways of TLR4 activation that have not been explored until now. It is likely that, in the future, other microbial or endogenous TLR4 stimulators (like amino acid-containing lipid present in many Gram-negative bacteria [38, 39], but also gangliosides [40] or ceramides [41, 42], which all share common structural features with diC14-amidine) will be 


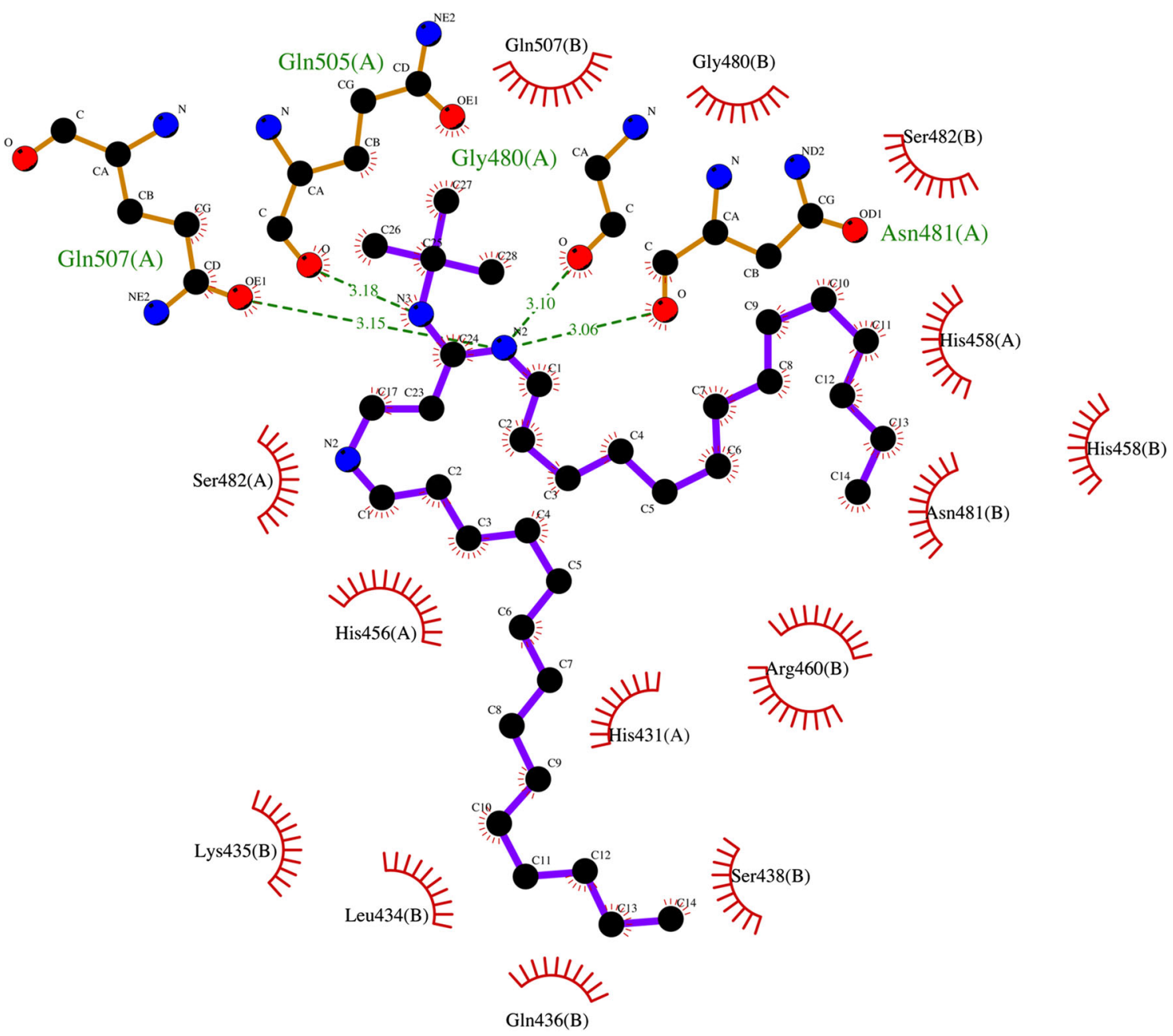

Fig. 5 Potential docking of diC14-amidine within the TLR4 dimer interface. Docking pause at $-5.2 \mathrm{kcal} / \mathrm{mol}$ involving hydrogen bonds with the side chain of Gln 507 and the main chain carbonyl groups of

demonstrated to be able to activate TLR4 through interaction with the TLR4 dimerization interface, expanding the possible ligands of TLR4 to non-MD-2-binding lipids. Finally, this new recognition interface might also be involved in the recognition of nanoparticles by the innate immune system. Indeed, diC14-amidine liposomes are not the only example of lipid-based nanoparticles, or more generally of engineered nanoparticles, that activate Toll-like receptor 4 [26, 43-46]. Our work can therefore contribute to a deeper knowledge of the effects of engineered nanoparticles on the immune system, a necessary step for their safer use in nanomedicine and for an improved therapeutic efficacy.

In addition, our data also demonstrate that diC14-amidine does not require CD14 to activate MyD88-dependent pathways. Similar CD14-independent behaviour has been reported for two synthetic lipid A derivatives: MPL (Monophosphoryl Lipid A) and CRX-527 and for the rough form of LPS (LPS lacking the full-length $\mathrm{O}$-antigen
Gly 480, Asn 481, and Gln 505, as well as a number of hydrophobic contacts at the TLR4 dimer interface. Figure generated by LigPlot [29]

chains-see Fig. S1) [47-50]. CD14 ligand carrier role has been established beyond doubt and is involved in the presentation of LPS to TLR4/MD-2 for initiating the MyD88dependent pathway [51]. The lack of the long polysaccharide chains in these LPS derivatives probably allows their better incorporation into and higher mobility in the mammalian cell membrane, providing a better access to protein receptors [48]. Therefore, the ability of diC14amidine, with its small hydrophilic headgroup, to be inserted into cell membranes after fusion [52, 53] offers a straightforward explanation for why this ligand does not require CD14 for inducing the MyD88-dependent pathway.

Finally, while it was generally accepted that CD14 was required for the LPS-induced endocytosis of TLR4 [54] which is considered necessary to enable the activation of the TRIF-dependent pathway [51, 55], our results demonstrate that CD14 is also not required to trigger the TRIFdependent pathway induced by diC14-amidine, in contrast 
to stimulation by rough LPS, MPL and CRX-527 [47-50]. Such behaviour has been described for LPS-coated latex beads [54] or LPS-formulated liposomes [56], which showed enhanced LPS endocytosis in the absence of CD14 (as compared to free LPS) thus confirming the importance of endocytosis for the TRIF-dependent signalling pathway $[54,55]$. However, those LPS formulations were found unable to activate the My88-dependent signalling pathway from inside the endosomes [56]. The uniqueness of diC14amidine, as compared to other known TLR4 ligands, to activate both signalling pathways in the absence of CD14, may therefore be related to the fact that diC14-amidine liposomes enter the cells via both endocytosis and fusion processes [53, 57].

In conclusion, here we show that the TLR4 agonist activity of the cationic lipid nanocarrier diC14-amidine is primarily dependent on its interaction with TLR4 by a mechanism likely similar to that proposed for nickel and cobalt ions. Important residues located at the $\mathrm{N}$ - and C-terminal edges of the TLR4/TLR4* dimerization interface are distinct from those reported for LPS binding and explain why two molecules as structurally different as diC14-amidine and LPS are both TLR4 activators. This may represent a new lead in developing compounds targeting these interactions in TLR4 without affecting the other functionalities of the receptor, in particular the recognition of conventional ligands.

Acknowledgments C.L. is a IEF Marie Curie Action Research Fellow (TLR4-CAT PIEF-GA-2012-326481) and would like to thank the Wiener-Anspach Foundation for financial support. K.L.I. was supported by a HBLB Veterinary Research Training scholarship. This work was supported by program grant support from the Wellcome Trust and the MRC to N.J.G and C.E.B.

\section{References}

1. Gay NJ, Gangloff M (2007) Structure and function of Toll receptors and their ligands. Annu Rev Biochem 76:141-165. doi:10.1146/annurev.biochem.76.060305.151318

2. Bryant CE, Spring DR, Gangloff M, Gay NJ (2010) The molecular basis of the host response to lipopolysaccharide. Nat Rev Microbiol 8:8-14. doi:10.1038/nrmicro2266

3. Park BS, Song DH, Kim HM, Choi BS, Lee H, Lee JO (2009) The structural basis of lipopolysaccharide recognition by the TLR4-MD-2 complex. Nature 458:1191-1195. doi:10.1038/ nature 07830

4. Ohto U, Fukase K, Miyake K, Satow Y (2007) Crystal structures of human MD-2 and its complex with antiendotoxic lipid IVa. Science 316:1632-1634. doi:10.1126/science.1139111

5. Poltorak A, Ricciardi-Castagnoli P, Citterio S, Beutler B (2000) Physical contact between lipopolysaccharide and toll-like receptor 4 revealed by genetic complementation. Proc Natl Acad Sci USA 97:2163-2167. doi:10.1073/pnas.040565397

6. DeMarco ML, Woods RJ (2011) From agonist to antagonist: structure and dynamics of innate immune glycoprotein MD-2 upon recognition of variably acylated bacterial endotoxins. Mol Immunol 49:124-133. doi:10.1016/j.molimm.2011.08.003

7. Chilton PM, Embry CA, Mitchell TC (2012) Effects of differences in lipid a structure on TLR4 pro-inflammatory signaling and inflammasome activation. Front Immunol 3:154. doi:10. 3389/fimmu.2012.00154

8. Oblak A, Jerala R (2014) The molecular mechanism of speciesspecific recognition of lipopolysaccharides by the MD-2/TLR4 receptor complex. Mol Immunol. doi:10.1016/j.molimm.2014.06. 034

9. Lien E, Means TK, Heine H, Yoshimura A, Kusumoto S, Fukase K, Fenton MJ, Oikawa M, Qureshi N, Monks B, Finberg RW, Ingalls RR, Golenbock DT (2000) Toll-like receptor 4 imparts ligand-specific recognition of bacterial lipopolysaccharide. J Clin. Invest 105:497-504. doi:10.1172/JCI8541

10. Bryant CE, Monie TP (2012) Mice, men and the relatives: crossspecies studies underpin innate immunity. Open Biol 2:120015. doi:10.1098/rsob.120015

11. Lohmann KL, Vandenplas M, Barton MH, Moore JN (2003) Lipopolysaccharide from Rhodobacter sphaeroides is an agonist in equine cells. J Endotoxin Res 9:33-37. doi:10.1177/ 09680519030090010301

12. Lohmann KL, Vandenplas ML, Barton MH, Bryant CE, Moore JN (2007) The equine TLR4/MD-2 complex mediates recognition of lipopolysaccharide from Rhodobacter sphaeroides as an agonist. J Endotoxin Res 13:235-242. doi:10.1177/0968051907083193

13. Irvine KL, Gangloff M, Walsh CM, Spring DR, Gay NJ, Bryant CE (2014) Identification of key residues that confer Rhodobacter sphaeroides LPS activity at horse TLR4/MD-2. PLoS One 9:e98776. doi:10.1371/journal.pone.0098776

14. Akashi S, Nagai Y, Ogata H, Oikawa M, Fukase K, Kusumoto S, Kawasaki K, Nishijima M, Hayashi S, Kimoto M, Miyake K (2001) Human MD-2 confers on mouse Toll-like receptor 4 species-specific lipopolysaccharide recognition. Int Immunol 13:1595-1599. doi:10.1093/intimm/13.12.1595

15. Sauter KS, Brcic M, Franchini M, Jungi TW (2007) Stable transduction of bovine TLR4 and bovine MD-2 into LPS-nonresponsive cells and soluble CD14 promote the ability to respond to LPS. Vet Immunol Immunopathol 118:92-104. doi:10.1016/j. vetimm.2007.04.017

16. Walsh C, Gangloff M, Monie T, Smyth T, Wei B, McKinley TJ, Maskell D, Gay N, Bryant C (2008) Elucidation of the MD-2/ TLR4 interface required for signaling by lipid IVa. J Immunol 181:1245-1254. doi:10.4049/jimmunol.181.2.1245

17. Ohto U, Fukase K, Miyake K, Shimizu T (2012) Structural basis of species-specific endotoxin sensing by innate immune receptor TLR4/MD-2. Proc Natl Acad Sci USA 109:7421-7426. doi:10. 1073/pnas.1201193109

18. Hajjar AM, Ernst RK, Tsai JH, Wilson CB, Miller SI (2002) Human Toll-like receptor 4 recognizes host-specific LPS modifications. Nat Immunol 3:354-359. doi:10.1038/ni777

19. Meng J, Drolet JR, Monks BG, Golenbock DT (2010) MD-2 residues tyrosine 42 , arginine 69 , aspartic acid 122 , and leucine 125 provide species specificity for lipid IVA. J Biol Chem 285:27935-27943. doi:10.1074/jbc.M110.134668

20. Meng J, Lien E, Golenbock DT (2010) MD-2-mediated ionic interactions between lipid A and TLR4 are essential for receptor activation. J Biol Chem 285:8695-8702. doi:10.1074/jbc.M109. 075127

21. Ruysschaert JM, El OA, Willeaume V, Huez G, Fuks R, Vandenbranden M, Di SP (1994) A novel cationic amphiphile for transfection of mammalian cells. Biochem Biophys Res Commun 203:1622-1628. doi:10.1006/bbrc.1994.2372

22. Elouahabi A, Flamand V, Ozkan S, Paulart F, Vandenbranden M, Goldman M, Ruysschaert JM (2003) Free cationic liposomes inhibit the inflammatory response to cationic lipid-DNA complex 
injected intravenously and enhance its transfection efficiency. Mol Ther 7:81-88. doi:10.1016/S1525-0016(02)00032-1

23. Tanaka T, Legat A, Adam E, Steuve J, Gatot JS, Vandenbranden M, Ulianov L, Lonez C, Ruysschaert JM, Muraille E, Tuynder M, Goldman M, Jacquet A (2008) DiC14-amidine cationic liposomes stimulate myeloid dendritic cells through Toll-like receptor 4. Eur J Immunol 38:1351-1357. doi:10.1002/eji.200737998

24. Lonez C, Vandenbranden M, Ruysschaert JM (2008) Cationic liposomal lipids: from gene carriers to cell signaling. Prog Lipid Res 47:340-347. doi:10.1016/j.plipres.2008.03.002

25. Lonez C, Lensink MF, Vandenbranden M, Ruysschaert JM (2009) Cationic lipids activate cellular cascades. Which receptors are involved? Biochim Biophys Acta 1790:425-430. doi:10. 1016/j.bbagen.2009.02.015

26. Lonez C, Vandenbranden M, Ruysschaert JM (2012) Cationic lipids activate intracellular signaling pathways. Adv Drug Deliv Rev 64:1749-1758. doi:10.1016/j.addr.2012.05.009

27. Trott O, Olson AJ (2010) AutoDock Vina: improving the speed and accuracy of docking with a new scoring function, efficient optimization, and multithreading. J Comput Chem 31:455-461. doi: $10.1002 /$ jcc. 21334

28. Pettersen EF, Goddard TD, Huang CC, Couch GS, Greenblatt DM, Meng EC, Ferrin TE (2004) UCSF Chimera-a visualization system for exploratory research and analysis. J Comput Chem 25:1605-1612. doi:10.1002/jcc. 20084

29. Wallace AC, Laskowski RA, Thornton JM (1995) LIGPLOT: a program to generate schematic diagrams of protein-ligand interactions. Protein Eng 8:127-134. doi:10.1093/protein/8.2.127

30. Schmidt M, Raghavan B, Muller V, Vogl T, Fejer G, Tchaptchet S, Keck S, Kalis C, Nielsen PJ, Galanos C, Roth J, Skerra A, Martin SF, Freudenberg MA, Goebeler M (2010) Crucial role for human Toll-like receptor 4 in the development of contact allergy to nickel. Nat Immunol 11:814-819. doi:10.1038/ni.1919

31. Oblak A, Pohar J, Jerala R (2015) MD-2 determinants of nickel and cobalt-mediated activation of human TLR4. PLoS One 10:e0120583. doi:10.1371/journal.pone.0120583

32. Inohara N, Nunez G (2002) ML-a conserved domain involved in innate immunity and lipid metabolism. Trends Biochem Sci 27:219-221. doi:10.1016/S0968-0004(02)02084-4

33. Dundas J, Ouyang Z, Tseng J, Binkowski A, Turpaz Y, Liang J (2006) CASTp: computed atlas of surface topography of proteins with structural and topographical mapping of functionally annotated residues. Nucleic Acids Res 34:W116-W118. doi:10.1093/ nar/gk1282

34. Jin MS, Kim SE, Heo JY, Lee ME, Kim HM, Paik SG, Lee H, Lee JO (2007) Crystal structure of the TLR1-TLR2 heterodimer induced by binding of a tri-acylated lipopeptide. Cell 130:1071-1082. doi:10.1016/j.cell.2007.09.008

35. Kang JY, Nan X, Jin MS, Youn SJ, Ryu YH, Mah S, Han SH, Lee H, Paik SG, Lee JO (2009) Recognition of lipopeptide patterns by Toll-like receptor 2-Toll-like receptor 6 heterodimer. Immunity 31:873-884. doi:10.1016/j.immuni.2009.09.018

36. Nattermann J, Du X, Wei Y, Shevchenko D, Beutler B (2000) Endotoxin-mimetic effect of antibodies against Toll-like receptor 4. J Endotoxin Res 6:257-264. doi:10.1177/09680519000060030701

37. Latz E, Visintin A, Lien E, Fitzgerald KA, Monks BG, KurtJones EA, Golenbock DT, Espevik T (2002) Lipopolysaccharide rapidly traffics to and from the Golgi apparatus with the toll-like receptor 4-MD-2-CD14 complex in a process that is distinct from the initiation of signal transduction. $\mathrm{J}$ Biol Chem 277:47834-47843. doi:10.1074/jbc.M207873200

38. Kawai Y, Takasuka N, Inoue K, Akagawa K, Nishijima M (2000) Ornithine-containing lipids stimulate CD14-dependent TNF-alpha production from murine macrophage-like J774.1 and RAW 264.7 cells. FEMS Immunol Med Microbiol 28:197-203. doi:10. 1111/j.1574-695X.2000.tb01477.x
39. Gomi K, Kawasaki K, Kawai Y, Shiozaki M, Nishijima M (2002) Toll-like receptor 4-MD-2 complex mediates the signal transduction induced by flavolipin, an amino acid-containing lipid unique to Flavobacterium meningosepticum. J Immunol 168:2939-2943. doi:10.4049/jimmunol.168.6.2939

40. Jou I, Lee JH, Park SY, Yoon HJ, Joe EH, Park EJ (2006) Gangliosides trigger inflammatory responses via TLR4 in brain glia. Am J Pathol 168:1619-1630. doi:10.2353/ajpath.2006.050924

41. Fischer H, Ellstrom P, Ekstrom K, Gustafsson L, Gustafsson M, Svanborg C (2007) Ceramide as a TLR4 agonist; a putative signalling intermediate between sphingolipid receptors for microbial ligands and TLR4. Cell Microbiol 9:1239-1251. doi:10. 1111/j.1462-5822.2006.00867.x

42. Hung LC, Lin CC, Hung SK, Wu BC, Jan MD, Liou SH, Fu SL (2007) A synthetic analog of alpha-galactosylceramide induces macrophage activation via the TLR4-signaling pathways. Biochem Pharmacol 73:1957-1970. doi:10.1016/j.bcp.2007.03.006

43. Kedmi R, Ben-Arie N, Peer D (2010) The systemic toxicity of positively charged lipid nanoparticles and the role of Toll-like receptor 4 in immune activation. Biomaterials 31:6867-6875. doi:10.1016/j.biomaterials.2010.05.027

44. Uto T, Akagi T, Yoshinaga K, Toyama M, Akashi M, Baba M (2011) The induction of innate and adaptive immunity by biodegradable poly(gamma-glutamic acid) nanoparticles via a TLR4 and MyD88 signaling pathway. Biomaterials 32:5206-5212. doi:10.1016/j.biomaterials.2011.03.052

45. Mano SS, Kanehira K, Taniguchi A (2013) Comparison of cellular uptake and inflammatory response via toll-like receptor 4 to lipopolysaccharide and titanium dioxide nanoparticles. Int J Mol Sci 14:13154-13170. doi:10.3390/ijms140713154

46. Qu G, Liu S, Zhang S, Wang L, Wang X, Sun B, Yin N, Gao X, Xia T, Chen JJ, Jiang GB (2013) Graphene oxide induces toll-like receptor 4 (TLR4)-dependent necrosis in macrophages. ACS Nano 7:5732-5745. doi:10.1021/nn402330b

47. Jiang Z, Georgel P, Du X, Shamel L, Sovath S, Mudd S, Huber M, Kalis C, Keck S, Galanos C, Freudenberg M, Beutler B (2005) CD14 is required for MyD88-independent LPS signaling. Nat Immunol 6:565-570. doi:10.1038/ni1207

48. Huber M, Kalis C, Keck S, Jiang Z, Georgel P, Du X, Shamel L, Sovath S, Mudd S, Beutler B, Galanos C, Freudenberg MA (2006) R-form LPS, the master key to the activation of TLR4/ MD-2-positive cells. Eur J Immunol 36:701-711. doi:10.1002/eji. 200535593

49. Legat A, Thomas S, Hermand P, Van Mechelen M, Goldman M, De Wit D (2010) CD14-independent responses induced by a synthetic lipid A mimetic. Eur J Immunol 40:797-802. doi:10. 1002/eji.200939992

50. Tanimura N, Saitoh SI, Ohto U, Akashi-Takamura S, Fujimoto Y, Fukase K, Shimizu T, Miyake K (2014) The attenuated inflammation of MPL is due to the lack of CD14-dependent tight dimerization of the TLR4/MD2 complex at the plasma membrane. Int Immunol. doi:10.1093/intimm/dxt071

51. Ostuni R, Zanoni I, Granucci F (2010) Deciphering the complexity of Toll-like receptor signaling. Cell Mol Life Sci 67:4109-4134. doi:10.1007/s00018-010-0464-x

52. Oliveira TR, Duarte EL, Lamy MT, Vandenbranden M, Ruysschaert JM, Lonez C (2012) Temperature-dependence of cationic lipid bilayer intermixing: possible role of interdigitation. Langmuir 28:4640-4647. doi:10.1021/la3001569

53. Lonez C, Lensink MF, Kleiren E, Vanderwinden JM, Ruysschaert JM, Vandenbranden M (2010) Fusogenic activity of cationic lipids and lipid shape distribution. Cell Mol Life Sci 67:483-494. doi:10.1007/s00018-009-0197-x

54. Zanoni I, Ostuni R, Marek LR, Barresi S, Barbalat R, Barton GM, Granucci F, Kagan JC (2011) CD14 controls the LPS-induced endocytosis of Toll-like receptor 4. Cell 147:868-880 
55. Kagan JC, Su T, Horng T, Chow A, Akira S, Medzhitov R (2008) TRAM couples endocytosis of Toll-like receptor 4 to the induction of interferon-beta. Nat Immunol 9:361-368. doi:10.1038/ ni1569

56. Watanabe S, Kumazawa Y, Inoue J (2013) Liposomal lipopolysaccharide initiates TRIF-dependent signaling pathway independent of CD14. PLoS One 8:e60078. doi:10.1371/journal. pone. 0060078
57. El Ouahabi A, Thiry M, Pector V, Fuks R, Ruysschaert JM, Vandenbranden M (1997) The role of endosome destabilizing activity in the gene transfer process mediated by cationic lipids. FEBS Lett 414:187-192. doi:10.1016/S0014-5793(97)00973-3 